\title{
Effect of Single Layer Centrifugation Porcicoll (70\%, 80\% and 90\%) or supplementation with reduced glutathione, seminal plasma and bovine serum albumin on frozen-thawed boar sperm
}

\author{
I. Crespo-F lez I⿳, A. Casta eda ${ }^{\mathrm{a}}$, D.I. S nchez ${ }^{\mathrm{b}}$, E. Fern ndez-Alegre ${ }^{\mathrm{a}}$, M. lvarez-Rodr guez, ,

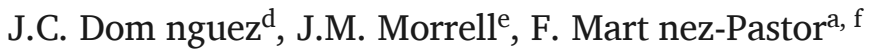

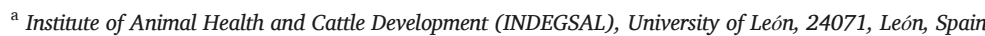 \\ ${ }^{\mathrm{b}}$ Institute of Biomedicine (IBIOMED), University of León, 24071, León, Spain \\ ${ }^{c}$ Department of Clinical and Experimental Medicine (IKE). BKH, Obstetrics and Gynecology, Linköping University, 58183, Linköping, Sweden \\ ${ }^{\mathrm{d}}$ Department of Animal Medicine, Surgery and Anatomy (Animal Medicine and Surgery), University of León, 24071, León, Spain \\ e Division of Reproduction, Swedish University of Agricultural Sciences, 75007, Uppsala, Sweden \\ ${ }^{\mathrm{f}}$ Dept. of Molecular Biology (Cell Biology), University of León, León, Spain
}

\section{A R T I C LE INFO}

\section{Keywords:}

Bovine serum albumin

Pig

Semen cryopreservation

Seminal plasma

Reduced glutathione

Single-layer centrifugation

\begin{abstract}
A B S T R A C T
Selecting the optimal sperm population is essential for success with reproductive techniques. Porcicoll (formerly Androcoll-P) is a colloid formulation for selection of high-quality boar spermatozoa by single layer centrifugation (SLC). To date, most studies have been carried out with fresh semen and large volumes. We carried out 2 experiments to test the use of Porcicoll for thawed boar semen in small volumes. In Experiment 1, cryopreserved semen doses were thawed, split in 200- 1 aliquots and layered on $1 \mathrm{~mL}$ of Porcicoll $70 \%, 80 \%$ or $90 \%$, or buffer without colloid. We assessed sperm recovery (the proportion of the loading dose that appeared in the pellet, \%), and the physiology of the selected spermatozoa (flow cytometry: Viability, apoptotic changes, capacitation, mitochondrial activity, intracellular reactive oxygen species). The most suitable proportion was Porcicoll 80\%, allowing acceptable sperm recovery (16.9 $4.2 \%$, compared to $70 \%$ $(35.4 \% 3.0, \mathrm{p}<0.001)$ and $90 \%(8.2 \% \quad 3.0, \mathrm{P}=0.001)$, and improved quality (mitochondrial activity: Porcicoll 80\%: $77.7 \quad 1 \%$ vs Control: $60.3 \quad 0.7 \%$, P < 0.05). In Experiment 2, we compared 3 supplements to Porcicoll 80\%: $500 \mathrm{mM}$ reduced glutathione (GSH), 20\% seminal plasma (SP) and $0.5 \%$ bovine serum albumin (BSA). Supplementation with GSH or BSA did not cause relevant changes relative to Control. In contrast, SP induced membrane and acrosomal changes resembling capacitation, which might preclude its use in some applications, and decreased recovery $(5.5 \% \quad 1.9$ vs. $24.3 \% \quad 1.2$ Control; $\mathrm{P}<0.001)$. However, it could be useful prior to other applications such as in vitro fertilisation. Overall, Porcicoll is an effective colloid for isolating a high-quality population from thawed boar sperm, $80 \%$ being a balanced option for good recovery and high quality. Supplements could be useful depending on the proposed use of the spermatozoa.
\end{abstract}

Corresponding author at: Faculty of Medicine and Health Sciences, Department of Clinical and Experimental Medicine (IKE), Obstetrics and Gynecology, Link ping University, SE-58185, Link ping, Sweden.

Email address: manuel.alvarez-rodriguez@liu.se (M. Álvarez-Rodríguez) 


\section{Introduction}

The swine industry is a dynamic and economically relevant activity worldwide. Recently it has experienced significant changes mainly due to genetic selection (Roca et al., 2016), with artificial insemination (AI) playing a significant role. However, the use of cryopreserved semen is still marginal, despite many advantages, (Yeste, 2015).

Boar spermatozoa are vulnerable to cryopreservation, which has motivated the quest to develop methods for selecting functional spermatozoa. Colloid centrifugation has been used in different species to improving the quality of spermatozoa, either before cryopreservation (Martinez-Alborcia et al., 2012) or after (Dorado et al., 2013). One suggestion is to use density gradient centrifugation (DGC) (Noguchi et al., 2015), since optimal spermatozoa may be denser than others. However, DGC can be time-consuming because of the preparation of the colloid column (Morrell and Rodriguez-Martinez, 2011), and thus single-layer centrifugation (SLC), using only one layer of a silane-coated silica colloid, has been proposed as a practical alternative. This technique has the advantages of simplicity, time-saving and no toxicity (Morrell et al., 2009). The first report of SLC with boar semen used Porcicoll (formerly known as Androcoll-P), a colloid optimized for this species (Morrell et al., 2009). This methodology has been scaled-up to process the large volumes of ejaculates typical for boar semen (van Wienen et al., 2011), for pathogen removal (Blomqvist et al., 2011) and for improving semen cryopreservation (Martinez-Alborcia et al., 2012, 2013).

However, there are no studies testing the effect of the post-thawing application of SLC on boar semen, dealing with the lower semen volumes resulting from thawing one straw of frozen semen.

Various substances have been shown to have a positive effect on boar spermatozoa in previous studies: reduced glutathione (GSH) used in cryopreservation and after thawing (Yeste et al., 2014); seminal plasma, playing an active role in maintaining of sperm physiology (Garcia et al., 2010; Fern ndez-Gago et al., 2013, 2016); and BSA, as cell membrane protector (Zhang et al., 2015).

Thus, the aim of the present study was to evaluate the ability of SLC with Porcicoll to select a high-quality population from cryopreserved boar semen. First, different concentrations of Porcicoll were tested with thawed boar semen, to assess their ability to select a sperm subpopulation with good functionality. Moreover, the effect of supplementing the colloid with different substances was studied.

\section{Materials and methods}

\subsection{Experimental design}

Commercial cryopreserved semen doses were obtained from Topigs-Norsvin Spain and only ejaculates with at least $70 \%$ motile and $75 \%$ morphologically normal spermatozoa immediately after collection were used to prepare doses. Therefore, this study did not involve live animals. Five experimental sessions were conducted in each of two experiments. In each session, a pool was prepared from 3 good-quality doses thawed at $37 \mathrm{C}$ for $30 \mathrm{~s}$ in a circulating water bath. Immediately after thawing, sample centrifugation with Porcicoll was carried out in 1.5-ml microcentrifuge tubes The sperm concentration of the pooled samples was adjusted to $150 \quad 10^{6}$ $\mathrm{mL}^{-1}$ with PBS and 2001 of this sperm suspension were carefully layered on top of the Porcicoll. The tubes were centrifuged at $300 \mathrm{~g}$ for $10 \mathrm{~min}$ at $25 \mathrm{C}$. The pellet was washed with $5001 \mathrm{of}$ PBS-BSA (0.5\% BSA, $600 \mathrm{~g}$ for $3 \mathrm{~min})$ and resuspended with 1501 of PBS-BSA. Sperm quality was analyzed before and after SLC and after a post-SLC incubation of $1 \mathrm{~h}$ at $37 \mathrm{C}$.

In the first experiment, we tested $1 \mathrm{~mL}$ Porcicoll at 70\%, 80\% and 90\%. Control samples were carefully layered on top of $1 \mathrm{~mL}$ PBS, to determine the effect of the centrifugation itself on post-thaw sperm quality. The preparations were centrifuged and processed as described above. In this experiment, the spermatozoa retained at the interface between the colloid and semen were also analyzed, to determine the number of good-quality spermatozoa prevented from passing into the colloid.

In the second experiment, Porcicoll 80\% was supplemented with either $500 \mathrm{mM}$ glutathione (GSH), 20\% seminal plasma (SP, substituting SP for the buffer when preparing Porcicoll $80 \%$ ) or $0.5 \%$ bovine serum albumin (BSA). A control tube contained Porcicoll $80 \%$ with no supplements. Tubes were centrifuged and processed as indicated.

\subsection{Reagents and media}

General reagents were purchased from Sigma (St. Louis, MO, EE UU.). Fluorescence probes were bought from Invitrogen (Carlsbad, CA, EE UU.). Stock solutions were prepared in deionized water (propidium iodide, 1.5 mM; Hoechst 33342, 9 mM; PNA FITC, 1 mg/ ml) or DMSO (YO PRO 1, Mitotracker deep red, Merocyanine 540, MitoSOX, CM $\mathrm{H}_{2}$ DCFDA, $1 \mathrm{mM}$ ), and stored at - 20 C. Porcicoll, adapted for boar semen, and its specific dilution buffer were supplied by Prof. J.M. Morrell at the Swedish University of Agricultural Sciences (SLU).

\subsection{Semen collection and preservation}

Semen doses were obtained from the Technological Centre of Artificial Insemination (Topigs-Norsvin Spain, Campo de Villavidel, Le n, Spain), according to the technique described by Pel ez et al. (Pel ez et al., 2006). Boar semen (Landrace breed) was extended in lactose-egg yolk (3\% glycerol) at $10^{9} \mathrm{~mL}^{-1}$ and packaged at $5 \mathrm{C}$ in $0.25 \mathrm{~mL}$ straws. Straws were frozen in a programmable 
freezer. (Digitcool 5300 ZB 250; IMV, L'Aigle Cedex, France) The freezing rate was $-3 \mathrm{C} / \mathrm{min}$ from +5 to -6 C, hold for 1 min, and at $-20 \mathrm{C} / \mathrm{min}$ from $-6 \mathrm{C}$ to $-100 \mathrm{C}$. Samples were then plunged into liquid nitrogen $(-196 \mathrm{C})$ for storage.

\subsection{Seminal plasma collection}

The SP was obtained from ejaculates of 11 boars (Duroc, Large White and Landrace) yielding good semen quality and prolificacy. The pooled ejaculates were submitted to a double centrifugation ( $800 \mathrm{~g}$ for $10 \mathrm{~min}$ at $25 \mathrm{C}$ ). After checking that no spermatozoa were present, the supernatant was aliquotted and stored at $-20 \mathrm{C}$.

\subsection{Sperm recovery assessment}

Sperm concentration was assessed in order to estimate the recovery in each SLC column. Samples were loaded in a B rker hemocytometer and analyzed using a computer-assisted sperm analyzer (CASA), consisting of an optical phase-contrast microscope (Eclipse 200, Nikon; Tokyo, Japan) with a Basler A302 fs digital camera (Basler Vision Technologies, Ahrensburg, Germany). Images were analyzed using the ISAS software (Proiser, Valencia, Spain). The recovery was calculated as [cells in the pellet]/[cells layered on the column] 100 .

\subsection{Flow cytometry}

Different combinations of the following fluorescent probes in PBS (0.5\% BSA) were used for assessing cell physiology (Mart nez-Pastor et al., 2010): YO PRO 1 (100 nM) for membrane permeability, propidium iodide (PI, 3 M) for membrane integrity, Mitotracker deep red (MTdr, $100 \mathrm{nM}$ ) for mitochondrial activity, PNA FITC (1 g/ml) for acrosomal status and Merocyanine 540 (M540, 2 M) for capacitation status. For the first experiment, we used the combinations YO PRO 1/PI/MTdr, PNA FITC/ PI and YO PRO 1/M540. We used the proportions of viable spermatozoa (YO PRO $1^{-} / \mathrm{PI}^{-}$), viable spermatozoa with increased membrane permeability (\%YO PRO $1^{+}$within the $\mathrm{PI}^{-}$population, termed apoptotic), spermatozoa with active mitochondria (YO PRO $1^{-} / \mathrm{MTdr}^{+}$), and viable capacitated spermatozoa (\%M540 ${ }^{+}$within the YO PRO $1^{-}$population) and acrosomal damaged (\%PNA FITC both $\mathrm{PI}^{+}$and $\mathrm{PI}^{-}$). In the second experiment, we further analyzed mitochondrial production of superoxide $\left(\left[\mathrm{O}_{2}{ }^{-}\right]_{\mathrm{m}}\right)$ and intracellular $\mathrm{H}_{2} \mathrm{O}_{2}\left(\left[\mathrm{H}_{2} \mathrm{O}_{2}\right]_{\mathrm{i}}\right)$, MitoSOX $(1 \mathrm{M})$ and CM $\mathrm{H}_{2}$ DCFDA $(1 \mathrm{M})$ respectively, with the combinations YO PRO 1/MitoSOX and $\mathrm{CM} \mathrm{H}_{2}$ DCFDA/PI. We recorded the proportions of viable spermatozoa with high $\left(\left[\mathrm{O}_{2}{ }^{-}\right]_{\mathrm{m}}\left(\% \mathrm{MitoSOX}^{+}\right.\right.$within the YO PRO $1^{-}$ population) and viable spermatozoa with high $\left(\left[\mathrm{H}_{2} \mathrm{O}_{2}\right]_{\mathrm{i}}\left(\% \mathrm{CM} \mathrm{H}_{2} \mathrm{DCFDA}^{+}\right.\right.$within the $\mathrm{PI}^{-}$population). Spermatozoa were added at $10^{6} \mathrm{~mL}^{-1}$ and incubated for $15 \mathrm{~min}$ at $37 \mathrm{C}$ in the dark. Hoechst 33342 (H342, $5 \mathrm{M}$ ) was added to all tubes for excluding debris from the fluorescence profiles.

Analyses were performed using a CyAn ADP cytometer (Beckman Coulter, Brea, CA, USA), equipped with three diode lasers (violet at $405 \mathrm{nM}$, blue at $488 \mathrm{nM}$ and red at $635 \mathrm{nM}$ ). The fluorescence was collected by photodetectors provided with filters $450 / 50$ (violet line, blue fluorescence: H342), 530/40 (blue line, green fluorescence: YO PRO 1, PNA-FITC, $\mathrm{H}_{2}$ DCFDA), $613 / 20$ (blue line, red fluorescence: PI, MitoSOX) and 665/20 (red line, red fluorescence: Mitotracker deep red). All parameters were visualized in a logarithmic scale. Spermatozoa were gated as $\mathrm{H}_{342}{ }^{+}$events, collecting at least 5000 spermatozoa. Data were processed using Weasel v3.1 (http: //www.frankbattye.com.au/Weasel/).

\subsection{Statistical analysis}

The statistical analyses were carried out with the R statistical package. Data were analyzed using linear mixed-effects models, with the treatments (Porcicoll concentrations or supplements) and incubation time as fixed effects, and the replicate as the grouping factor in the random part of the model. Pairwise comparisons were adjusted by Tukey s method. Results are presented as means SEM, and the threshold for significance was set at $\mathrm{P}<0.05$.

\section{Results}

\subsection{Experiment 1: evaluation of porcicoll concentrations in sperm recovery and quality}

The sperm recovery for the Porcicoll columns was $35.4 \% \quad 3.0,16.9 \% \quad 1.2$ and $8.2 \% \quad 3.0$ (70, 80 and $90 \%$, respectively) ( $\mathrm{P}<0.001$ for $70 \%$ vs. $90 \%, \mathrm{P}=0.001$ for $80 \%$ vs. $90 \%$ ).

Table 1 summarizes sperm quality after centrifugation. We did not detect a significant interaction between treatment and incubation time, and therefore we analyzed them as main effects. Sperm viability, mitochondrial activity and acrosomal integrity in the pellet (selected spermatozoa) increased when using Porcicoll at $80 \%$ or $90 \%$, whereas $70 \%$ did not improve them relative to Control (Control $70 \%<80 \%<90 \%$, P $<0.05$ ). The proportion of viable spermatozoa with capacitation-like features (M540 staining) was 
Table 1

Effect of Single Layer Centrifugation (SLC) with different proportions of Porcicoll (70\%, 80\% and 90\%) on sperm quality parameters.

\begin{tabular}{|c|c|c|c|c|c|c|c|c|c|}
\hline & \multicolumn{2}{|c|}{$\begin{array}{l}\text { Viability } \\
(\%)\end{array}$} & \multicolumn{2}{|c|}{$\begin{array}{l}\text { Apoptotic cells (ratio of } \\
\text { viable, \%) }\end{array}$} & \multicolumn{2}{|c|}{$\begin{array}{l}\text { Mitochondrial activity } \\
(\%)\end{array}$} & \multicolumn{2}{|c|}{$\begin{array}{l}\text { Damaged acrosomes } \\
(\%)\end{array}$} & $\begin{array}{l}\text { Capacitation (ratio of } \\
\text { viable, \%) }\end{array}$ \\
\hline \multicolumn{10}{|c|}{ Treatment } \\
\hline Control & 61.4 & $1.5^{\mathrm{a}}$ & 13.2 & 4.2 & 61.6 & $1.8^{\mathrm{a}}$ & 18.2 & $1.0^{\mathrm{a}}$ & $1.5 \quad 0.3^{\mathrm{a}}$ \\
\hline $\begin{array}{l}\text { Pellet } \\
70 \%\end{array}$ & 64.7 & $1.3^{\mathrm{a}}$ & 23.4 & 3.0 & 57.0 & $3.5^{\mathrm{a}}$ & 16.2 & $0.9^{\mathrm{a}}$ & $0.6 \quad 0.1^{b}$ \\
\hline $\begin{array}{l}\text { Pellet } \\
80 \%\end{array}$ & 77.7 & $0.7^{\mathrm{b}}$ & 19.8 & 1.4 & 73.2 & $2.5^{\mathrm{b}}$ & 9.2 & $0.6^{\mathrm{b}}$ & $0.5 \quad 0.1^{b}$ \\
\hline $\begin{array}{l}\text { Pellet } \\
90 \%\end{array}$ & 84.6 & $0.9^{c}$ & 14.1 & 3.3 & 82.2 & $1.6^{\mathrm{c}}$ & 6.4 & $0.9^{b}$ & $0.4 \quad 0.1^{b}$ \\
\hline Time & & & & & & & & & \\
\hline $0 \mathrm{~h}$ & 73.3 & 1.4 & 18.0 & 1.6 & 70.0 & 2.1 & 11.4 & 0.7 & $0.7 \quad 0.1$ \\
\hline $1 \mathrm{~h}$ & 71.0 & 1.4 & 17.3 & 1.6 & 67.0 & 2.1 & 13.6 & 0.7 & $0.8 \quad 0.1$ \\
\hline
\end{tabular}

Parameters analyzed: Viability, apoptotic cells, mitochondrial activity, acrosomal status and capacitation. (data from Control and Pellets at $0 \mathrm{~h}$ and $1 \mathrm{~h}$; the interaction treatment time was not significant, thus these two factors were analyzed as main effects). Results for the unprocessed sample (semen pool) and interfaces are shown in Table 2.

Results are the mean SEM. a c Different superscripts indicate significant differences between treatments (P $<0.05)$. Incubation did not affected the results significantly.

very low in all cases (only $1.5 \% \quad 0.3$ of viable spermatozoa were identified as M540 + in the Control), but SLC reduced these spermatozoa in all cases ( $0.5 \%$ ). Interestingly, incubation did not significantly affect any of these variables.

We also analyzed the quality of the spermatozoa recovered from the interface (unable to enter the colloid; Table 2). The effect of centrifugation alone (Control) was non-significant, comparing to the non-centrifuged pool. Viability and mitochondrial activity were significantly lower in all the interfaces than in the pellet or Control. The interface from Porcicoll $70 \%$ showed the lowest values for viability ( $\mathrm{P}<0.05$ comparing to $80 \%$ and $90 \%$ ), and the highest proportion of damaged acrosomes ( $<<0.05$ comparing to pool and Control). The occurrence of apoptotic markers did not change in the $80 \%$ and $90 \%$ interfaces.

\subsection{Experiment 2}

\subsubsection{Evaluation of supplements on the performance of SLC}

The GSH and BSA yielded similar recovery ratios to the Control (Porcicoll 80\%: 24.3\% 1.2; GSH: 24.8\% 2.1; BSA: $27.9 \% \quad 2.3$ ), but SP reduced it to $5.5 \% \quad 1.9$ ( $\mathrm{P}<0.001)$.

As in Experiment 1, treatments and incubation time did not interact significantly, being analyzed as main factors. The use of supplements during SLC caused small changes in sperm quality compared to the Control (Fig. 1). Only SP yielded a lower proportion of apoptotic cells (Fig. 1b) and a significantly higher viability and mitochondrial activity compared to GSH or BSA (Fig. 1a and c). This treatment also caused a reduction of viable cells with high $\left[\mathrm{H}_{2} \mathrm{O}_{2}\right]_{\mathrm{i}}$ (Fig. $2 \mathrm{~d}$; $\mathrm{P}<0.05$ compared to the Control and GSH). However, it also increased both acrosomal damage (Fig. 2a) and capacitation (Fig. 2b). The proportion of viable cells with high $\left[\mathrm{O}_{2}{ }^{\bullet-}\right]_{\mathrm{m}} \mathrm{was}$ low and not affected by the treatments (Fig. 2c).

Table 2

Comparison between the thawed-unprocessed sample (pool), the centrifuged Control and the spermatozoa recovered from the interfaces of the Porcicoll columns, after Single Layer Centrifugation (SLC) with different proportions of Porcicoll (70\%, $80 \%$ and $90 \%$ ).

\begin{tabular}{|c|c|c|c|c|c|c|c|c|c|}
\hline \multirow[b]{2}{*}{ Pool } & \multicolumn{2}{|c|}{$\begin{array}{l}\text { Viability } \\
(\%)\end{array}$} & \multirow{2}{*}{$\begin{array}{l}\text { Apoptotic cells (ratio of } \\
\text { viable, \%) }\end{array}$} & \multicolumn{2}{|c|}{$\begin{array}{l}\text { Mitochondrial activity } \\
(\%)\end{array}$} & \multicolumn{2}{|c|}{$\begin{array}{l}\text { Damaged acrosomes } \\
(\%)\end{array}$} & \multicolumn{2}{|c|}{$\begin{array}{l}\text { Capacitation (ratio of } \\
\text { viable, } \% \text { ) }\end{array}$} \\
\hline & 61.8 & $1.9^{\mathrm{a}}$ & & 57.2 & $4.3^{\mathrm{a}}$ & 20 & $2.8^{\mathrm{a}}$ & 4 & 1.7 \\
\hline Control & 59.9 & $2.8^{\mathrm{a}}$ & $12.7 \quad 4.7$ & 60.3 & $0.7^{\mathrm{a}}$ & 18.3 & $0.5^{\mathrm{a}}$ & 1.4 & 0.4 \\
\hline $\begin{array}{l}\text { Interface } \\
70 \%\end{array}$ & 38.6 & $1.3^{\mathrm{b}}$ & $15.3 \quad 2.3$ & 35.1 & $1.4^{\mathrm{b}}$ & 27.4 & $0.8^{\mathrm{b}}$ & 2.5 & 0.3 \\
\hline $\begin{array}{l}\text { Interface } \\
80 \%\end{array}$ & 48.3 & $1.5^{\mathrm{c}}$ & $21.5 \quad 1.6$ & 43.7 & $2.6^{\mathrm{b}}$ & 21.7 & $0.9^{\mathrm{ab}}$ & 2.6 & 0.5 \\
\hline $\begin{array}{l}\text { Interface } \\
90 \%\end{array}$ & 48.2 & $2.6^{c}$ & $25.7 \quad 5.1$ & 39.4 & $4.7^{\mathrm{b}}$ & 21.5 & $1.9^{\mathrm{ab}}$ & 2.1 & 0.3 \\
\hline
\end{tabular}

This table shown results just after SLC, since the pools and interfaces were not incubated afterwards. The results of the pellets were significantly different than those of the interface, except for the occurrence of apoptotic markers (see Table 1 for the Pellet values, averaged for 0 and $1 \mathrm{~h}$ ).

Parameters analyzed: Viability, apoptotic cells, mitochondrial activity, acrosomal status and capacitation.

Results are the mean SEM. ${ }^{\text {a-c }}$ Different superscripts indicate significant differences between treatments (P $\left.<0.05\right)$. Incubation did not affected the results significantly. 
(a) Viability

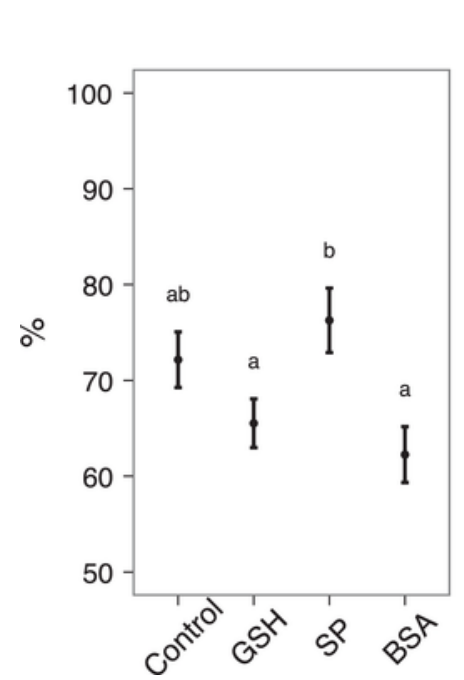

(b) Apoptotic cells (ratio of viable)

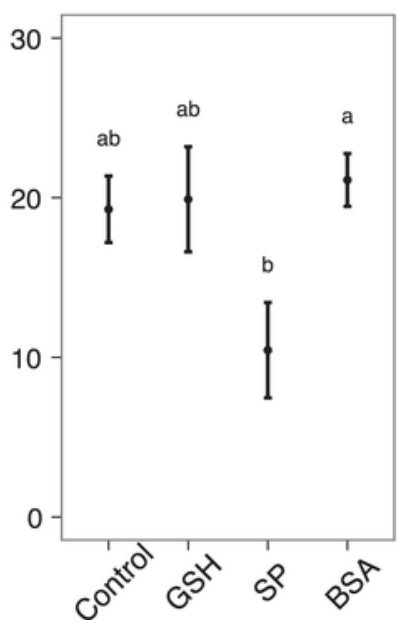

(c) Mitochondrial activity

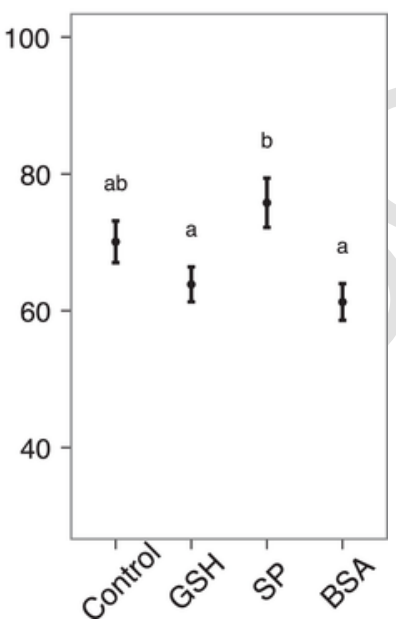

Fig. 1. Effects of Single Layer Centrifugation (SLC) with Porcicoll (80\%) supplemented with reduced glutathione (GSH), seminal plasma (SP) or bovine serum albumin (BSA), on viability (a), occurrence of apoptotic markers (b) and mitochondrial activity (c) of the spermatozoa recovered in the pellet. Results were adjusted for incubation time effects (the interaction treatment time was not significant; thus, these two factors were analyzed as main effects; see Table 3). Treatments with different letters differ $\mathrm{P}<0.05$.

(a) Damaged Acrosomes

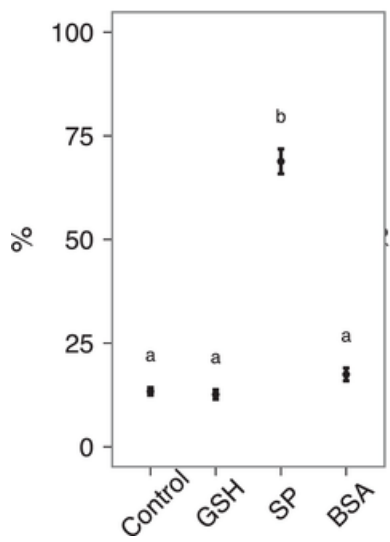

(b) Capacitation (ratio of viable)

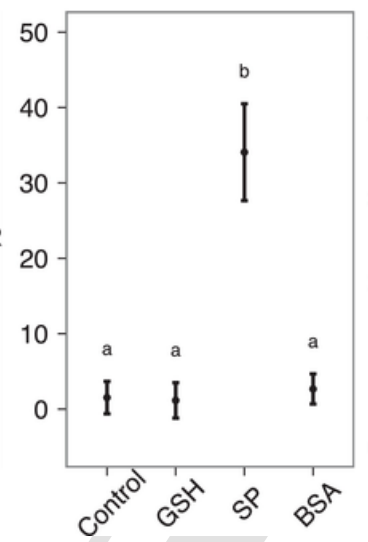

(c) High $\mathrm{O}_{2}$ (ratio of viable)

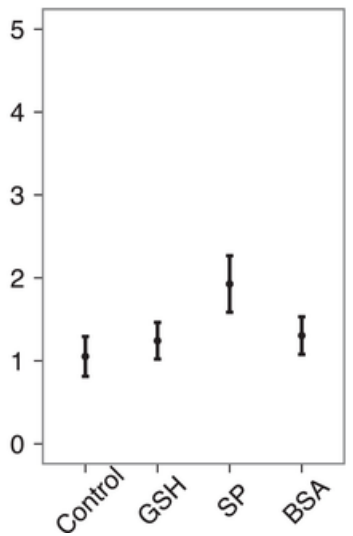

(d) High $\mathrm{H}_{2} \mathrm{O}_{2}$ (ratio of viable)

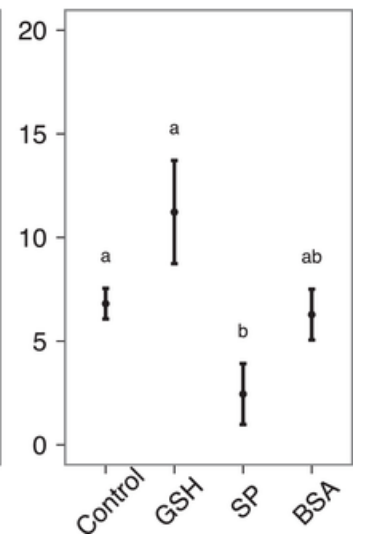

Fig. 2. Effects of Single Layer Centrifugation (SLC) with Porcicoll (80\%) supplemented with reduced glutathione (GSH), seminal plasma (SP) or bovine serum albumin (BSA), on the acrosomal status (a), capacitation (b) and the intracellular production of superoxide anion (c) or hydrogen peroxide (d) of the spermatozoa recovered in the pellet. Results were adjusted for incubation time effects (the interaction treatment time was not significant; thus, these two factors were analyzed as main effects; see Table 3). Treatments with different letters differ $\mathrm{P}<0.05$.

Incubation time in Experiment 2 had a significant effect on several variables (Table 3). It caused a significant, albeit small, drop in sperm viability and mitochondrial activity, while increasing the proportion of viable spermatozoa with apoptotic features or positive for $\left[\mathrm{H}_{2} \mathrm{O}_{2}\right]_{\mathrm{i}}$ production. Incubation did not significantly affect the proportion of spermatozoa with acrosomal damage, capacitation features or increased $\left[\mathrm{O}_{2}{ }^{--}\right]_{\mathrm{m}}$.

\section{Discussion}

Porcicoll has achieved good results with boar semen when used for SLC prior to freezing (Martinez-Alborcia et al., 2012, 2013). In this study, we have adapted it to the selection of spermatozoa from thawed boar semen. This is a very different scenario, in which not only volumes must be scaled down, but we also have to take into account that the cryopreservation process alters the sperm physiology. We tested how changing the proportion of colloid in the SLC and the presence of supplements could affect sperm recovery and quality. 
Table 3

Effect of incubation time in sperm parameters after Single Layer Centrifugation (SLC), on sperm quality parameters.

\begin{tabular}{|c|c|c|c|c|c|c|c|c|c|c|c|}
\hline & \multicolumn{2}{|c|}{$\begin{array}{l}\text { Viability } \\
(\%)\end{array}$} & \multicolumn{2}{|c|}{$\begin{array}{l}\text { Apoptotic cells } \\
\text { (ratio of viable, } \\
\% \text { ) }\end{array}$} & \multicolumn{2}{|c|}{$\begin{array}{l}\text { Mitochondrial } \\
\text { activity (\%) }\end{array}$} & \multicolumn{2}{|c|}{$\begin{array}{l}\text { Damaged } \\
\text { acrosomes } \\
(\%)\end{array}$} & $\begin{array}{l}\text { Capacitation } \\
\text { (ratio of viable, } \\
\% \text { ) }\end{array}$ & $\begin{array}{l}\text { High } \mathrm{O}_{2} \bullet- \\
\text { (ratio of } \\
\text { viable, \%) }\end{array}$ & $\begin{array}{l}{\left[\mathrm{H}_{2} \mathrm{O}_{2}\right]_{\mathrm{i}}} \\
\text { (ratio of } \\
\text { viable, \%) }\end{array}$ \\
\hline $0 \mathrm{~h}$ & 72.3 & 1.2 & 13.8 & 2.03 & 72.2 & 1.7 & 26.8 & 0.7 & $11.6 \quad 1.6$ & $1.6 \quad 0.2$ & $3.0 \quad 0.8$ \\
\hline $1 \mathrm{~h}$ & 65.8 & 1.2 & 21.6 & 2.03 & 63.3 & 1.7 & 29.3 & 0.7 & $8.2 \quad 1.6$ & $1.2 \quad 0.2$ & $10.4 \quad 0.8$ \\
\hline $\mathrm{P}$ & \multicolumn{2}{|c|}{0.020} & \multicolumn{2}{|c|}{0.006} & \multicolumn{2}{|c|}{0.003} & \multicolumn{2}{|c|}{0.107} & 0.620 & 0.168 & $<0.001$ \\
\hline
\end{tabular}

Parameters analyzed: viability, apoptotic cells, mitochondrial activity, acrosomal status, capacitation, and intracellular O2* and $\mathrm{H} 2 \mathrm{O} 2$ levels. Results are the mean SEM.

As expected, sperm recovery followed an inverse trend with the colloid concentration, due to the higher stringency of the selection. This was confirmed when studying the characteristics of both the pelleted spermatozoa and those retained at the interface. Fewer spermatozoa were recovered with $90 \%$ colloid than with $80 \%$ or $70 \%$ but their quality was even higher. This is the first study of this type carried out with thawed boar spermatozoa, and few studies on other species have been reported. Dorado et al. (2013) obtained a recovery of $63.3 \%$ with thawed dog semen using Androcoll-C (for dog spermatozoa), three times more than $80 \%$ Porcicoll. Thys et al. (2009) obtained 51.5\% recovery when using Androcoll-B for selecting thawed bull spermatozoa, while achieving improved motility and an IVF fertility ratio comparable to other selection methods. However, Jim nez-Rabad n et al. (2012) reported a recovery similar to $90 \%$ Porcicoll using Androcoll-B with thawed semen from goat. The differences between studies might be multifactorial, but the most important factor might be the species differences. Boar spermatozoa are well known for their vulnerability to cryopreservation (Martinez-Alborcia et al., 2013; Fern ndez-Gago et al., 2016), which might lead to fewer spermatozoa being of sufficient quality to be able to pass through the colloid into the sperm pellet.

Considering sperm viability, the increase achieved with Porcicoll $80 \%$ and $90 \%$ allow the recovery of a good quality pellet (Pinart et al., 1999). Mitochondrial activity, which is critical for sperm physiology, was also improved, and spermatozoa with damaged acrosomes were mostly removed from the sample. Our results are in agreement with studies in red deer (Anel-L pez et al., 2015) in which the authors reported an increase in sperm quality. However, their results were lower than those reported by us with 80\% Porcicoll, obtaining 67.3\% $\quad 3.1$ of viable sperm and 65.7\% $\quad 6.1$ of intact acrosomes for electroejaculated spermatozoa (and even lower results for epididymal samples). Dorado et al. (2013) reported 53.99\% $\quad 6.99$ viable and $22.40 \% \quad 0.03$ acrosome-damaged spermatozoa in dog, which are more similar to the results we obtained with $70 \%$ Porcicoll, and might be related to the higher recovery they obtained.

Capacitation is a crucial physiological process for mammalian spermatozoa, which must occur either in vivo during transit through female reproductive tract or in vitro in a defined media. Premature triggering of capacitation (e.g., due to cryopreservation) is detrimental (Watson, 2000). Using Merocyanine 540, we characterized a small capacitated population in the thawed samples. It is difficult to assess the actual importance of this population, but Porcicoll SLC almost completely removed it. This fact highlights the ability of Porcicoll to remove spermatozoa with putatively detrimental alterations, even though they are viable.

The quality of the spermatozoa at the interface can also help in assessing the efficiency of each Porcicoll proportion. An increasing concentration of the colloid increases its selectivity, and thus we might expect more apparently good-quality spermatozoa to be retained at the interface. Indeed, $80 \%$ and $90 \%$ of Porcicoll retained more viable spermatozoa than $70 \%$. However, these retained viable spermatozoa are potentially of lower quality, since there was also a higher retention of spermatozoa with apoptotic markers and damaged acrosomes. The importance of removing these abnormal spermatozoa from the insemination doses has been emphasized, due to the negative effects that they could exert over accompanying good-quality cells (Roca et al., 2013).

Thus, a higher proportion of Porcicoll was more efficient in obtaining a sperm population with improved physiological parameters, with little practical differences between $90 \%$ and $80 \%$. Therefore, $80 \%$ Porcicoll in small volumes could be the most practical choice for frozen-thawed boar spermatozoa, allowing a balance between a good recovery, removal of abnormal cells and obtaining a pellet of high-quality spermatozoa.

The second objective of this study was to test if adding different supplements to the Porcicoll could have a protective effect during centrifugation, increasing sperm recovery or quality. Many researchers have tested supplements for preventing or reversing the damage incurred by sperm storage, but none have dealt with adding supplements to the colloid itself.

The most important finding is the pronounced effect of SP on SLC results, as much for recovery as for quality. SP is a complex fluid, which is critical, not only for protecting and nourishing spermatozoa, but also for modulating their fertilizing capacity (Rodr guez-Mart nez et al., 1989). SP might alter sperm characteristics during centrifugation, explaining the higher cell retention by Porcicoll. Moreover, the physiology of the selected spermatozoa was also affected. The changes in membrane and acrosomal status after SLC with SP were the most noticeable and important results, since these events are related to sperm capacitation. Indeed, Fern ndez-Gago et al. (2013) found that 50\% SP increased the presence of capacitated spermatozoa in post-thaw spermatozoa. However, the application of SP often results in contradictory results depending on the type of sample (fresh, cooled, thawed or sorted) (Caballero et al., 2012). In our case, SLC might increase the capacitating effects of SP, leading to changes similar to those reported by Fern ndez-Gago et al. (2013). The increase in capacitated spermatozoa could be a drawback for some applications (e.g., sex sort- 
ing), but an advantage for others. For instance, the selected spermatozoa could be transferred to IVF (in vitro fertilisation) without the need for further capacitation steps.

We also assessed ROS generation, since the supplements could have an antioxidant effect. The low ROS levels reported by us might not be a problem in unprocessed samples, but centrifugation and pelleting are stressing processes, and they could transiently increase ROS production or sperm vulnerability (Dom nguez-Rebolledo et al., 2009). Nevertheless, we found that ROS changes were small, and they seemed to have little effect on the quality of our samples. Although cryopreservation increases ROS levels, this effect could be less important in pig comparing to other species (Yeste et al., 2013), and the endogenous ROS defense system in boar sperm might be very efficient (Guthrie et al., 2008). Moreover, in contrast to other studies where spermatozoa were exposed to supplements for several hours (BSA: Zhang et al., 2015; GSH: Yeste et al., 2014), our samples were exposed only during SLC (10 min), which might not be long enough for protective effects to be observed.

In conclusion, SLC with Porcicoll significantly improved the quality of cryopreserved boar semen. Porcicoll $80 \%$ was the most effective concentraiton of colloid for selecting a high-quality population while achieving good cell recovery. We also found that supplementing the colloid with different substances could be useful for modulating the characteristics of the selected sperm population, although the results varied. This modulation could be useful depending on the application, and SP may be an option if spermatozoa were to be immediately used for IVF.

\section{Conflict of interest}

Authors declare no conflict of interest in the present study. Professor J.M. Morrell is the inventor and patent holder for Porcicoll.

\section{Acknowledgements}

The authors thank Topigs-Norsvin Espa a (Madrid, Spain), and especially the staff at the Artificial Insemination Centre at Campo de Villavidel (Le n, Spain) for providing the semen doses; and Roc o Fern ndez, Marta Olmos, Judit Llorens and Karolina Pomorska for their help when setting up the experiments

\section{References}

Anel-L pez, L., Mart nez-Rodr guez, C., Soler, A.J., Fern ndez-Santos, M.R., Garde, J.J., Morrell, J.M., 2015. Use of Androcoll-S after thawing improves the quality of electroejaculated and epididymal sperm samples from red deer. Anim. Reprod. Sci. 158, 68-74.

Blomqvist, G., Persson, M., Wallgren, M., Wallgren, P., Morrell, J.M., 2011. Removal of virus from boar semen spiked with porcine circovirus type 2. Anim. Reprod. Sci. 126, 108-114.

Caballero, I., Parrilla, I., Almi ana, C., del Olmo, D., Roca, J., Mart nez, E.A., V zquez, J.M., 2012. Seminal plasma proteins as modulators of the sperm function and their application in sperm biotechnologies. Reprod. Domest. Anim. Zuchthyg. 47 (3), 12-21.

Dom nguez-Rebolledo, A.E., Fern ndez-Santos, M.R., Garc a-Alvarez, O., Maroto-Morales, A., Garde, J.J., Mart nez-Pastor, F., 2009. Washing increases the susceptibility to exogenous oxidative stress in red deer spermatozoa. Theriogenology 72, 1073-1084.

Dorado, J., G lvez, M.J., Morrell, J.M., Alcar z, L., Hidalgo, M., 2013. Use of single-layer centrifugation with Androcoll-C to enhance sperm quality in frozen-thawed dog semen. Theriogenology 80, 955-962.

Fern ndez-Gago, R., Dom nguez, J.C., Mart nez-Pastor, F., 2013. Seminal plasma applied post-thawing affects boar sperm physiology: a flow cytometry study. Theriogenology 80, 400-410.

Fern ndez-Gago, R., lvarez-Rodr guez, M., Alonso, M.E., Gonz lez, J.R., Alegre, B., Dom nguez, J.C., Mart nez-Pastor, F., 2016. Thawing boar semen in the presence of seminal plasma improves motility, modifies subpopulation patterns and reduces chromatin alterations. Reprod. Fertil. Dev. https://doi.org/10.1071/RD15530.

Garcia, J.C., Dominguez, J.C., Pena, F.J., Alegre, B., Gonzalez, R., Castro, M.J., Habing, G.G., Kirkwood, R.N., 2010. Thawing boar semen in the presence of seminal plasma: effects on sperm quality and fertility. Anim. Reprod. Sci. 119, 160-165.

Guthrie, H.D., Welch, G.R., Long, J.A., 2008. Mitochondrial function and reactive oxygen species action in relation to boar motility. Theriogenology 70, $1209-1215$.

Jim nez-Rabad n, P., Morrell, J.M., Johannisson, A., Ram n, M., Garc a- lvarez, O., Maroto-Morales, A., lvaro-Garc a, P.J., P rez-Guzm n, M.D., Fern ndez-Santos, M.R., Garde, J.J., Soler, A.J., 2012. Single layer centrifugation (SLC) improves sperm quality of cryopreserved Blanca-Celtib rica buck semen. Anim. Reprod. Sci. 136, 47-54.

Mart nez-Pastor, F., Mata-Campuzano, M., Alvarez-Rodr guez, M., Alvarez, M., Anel, L., de Paz, P., 2010. Probes and techniques for sperm evaluation by flow cytometry. Reprod. Domest. Anim. Zuchthyg. 45 (2), 67-78.

Martinez-Alborcia, M.J., Morrell, J.M., Parrilla, I., Barranco, I., V zquez, J.M., Martinez, E.A., Roca, J., 2012. Improvement of boar sperm cryosurvival by using single-layer colloid centrifugation prior freezing. Theriogenology 78, 1117-1125.

Martinez-Alborcia, M.J., Morrell, J.M., Gil, M.A., Barranco, I., Maside, C., Alkmin, D.V., Parrilla, I., Martinez, E.A., Roca, J., 2013. Suitability and effectiveness of single layer centrifugation using Androcoll-P in the cryopreservation protocol for boar spermatozoa. Anim. Reprod. Sci. 140, 173-179.

Morrell, J.M., Rodriguez-Martinez, H., 2011. Practical applications of sperm selection techniques as a tool for improving reproductive efficiency. Vet. Med. Int. https:// doi.org/10.4061/2011/894767.

Morrell, J.M., Saravia, F., Wienen, M., van Wallgren, M., Rodriguez-Martinez, H., 2009. Selection of boar spermatozoa using centrifugation on a glycidoxypropyltrimethoxysilane-coated silica colloid. J. Reprod. Dev. 55, 547-552.

Noguchi, M., Yoshioka, K., Hikono, H., Iwagami, G., Suzuki, C., Kikuchi, K., 2015. Centrifugation on Percoll density gradient enhances motility, membrane integrity and in vitro fertilizing ability of frozen-thawed boar sperm. Zygote Camb. Engl. 23, 68-75.

Pel ez, J., Breininger, E., Alegre, B., Pe a, F.J., Dom nguez, J.C., 2006. In vitro evaluation of the quality and fertilizing capacity of boar semen frozen in 0.25 ml straws. Reprod. Domest. Anim. Zuchthyg. 41, 153-161.

Pinart, E., Sancho, S., Briz, M.D., Bonet, S., Garc a, N., 1999. Characterization of the semen quality of postpuberal boars with spontaneous unilateral abdominal cryptorchidism on the right side. Anim. Reprod. Sci. 55, 269-278.

Roca, J., Martinez-Alborcia, M.J., Gil, M.A., Parrilla, I., Martinez, E.A., 2013. Dead spermatozoa in raw semen samples impair in vitro fertilization outcomes of frozen-thawed spermatozoa. Fertil. Steril. 100, 875-881.

Roca, J., Parrilla, I., Bolarin, A., Martinez, E.A., Rodriguez-Martinez, H., 2016. Will AI in pigs become more efficient?. Theriogenology 86, 187-193. 
Rodr guez-Mart nez, H., Kvist, U., Ernerudh, J., Sanz, L., Calvete, J.J., 1989. 2011. Seminal plasma proteins: what role do they play?. Am. J. Reprod. Immunol. N. Y. N 66 (1), 11-22.

Thys, M., Vandaele, L., Morrell, J.M., Mestach, J., Van Soom, A., Hoogewijs, M., Rodriguez-Martinez, H., 2009. In vitro fertilizing capacity of frozen-thawed bull spermatozoa selected by single-layer (glycidoxypropyltrimethoxysilane) silane-coated silica colloidal centrifugation. Reprod. Domest. Anim. Zuchthyg. 44, 390-394.

van Wienen, M., Johannisson, A., Wallgren, M., Parlevliet, J., Morrell, J.M., 2011. Single layer centrifugation with androcoll-P can Be scaled-Up to process larger volumes of boar semen. ISRN Vet. Sci. 1-8.

Watson, P.F., 2000. The causes of reduced fertility with cryopreserved semen. Anim. Reprod. Sci. 60 61, 481-492.

Yeste, M., Estrada, E., Casas, I., Bonet, S., Rodr guez-Gil, J.E., 2013. Good and bad freezability boar ejaculates differ in the integrity of nucleoprotein structure after freeze-thawing but not in ROS levels. Theriogenology 79, 929-939.

Yeste, M., Estrada, E., Pinart, E., Bonet, S., Mir , J., Rodr guez-Gil, J.E., 2014. The improving effect of reduced glutathione on boar sperm cryotolerance is related with the intrinsic ejaculate freezability. Cryobiology 68, 251-261.

Yeste, M., 2015. Recent advances in boar sperm cryopreservation: state of the art and current perspectives. Reprod. Domest. Anim. Zuchthyg. 50 (2), 71-79.

Zhang, X.G., Yan, G.J., Hong, J.Y., Su, Z.Z., Yang, G.S., Li, Q.W., Hu, J.H., 2015. Effects of bovine serum albumin on boar sperm quality during liquid storage at 17 C. Reprod. Domest. Anim. 50, 263-269. 